\title{
THE LOCAL EXTENSION OF NERVE FIBRES INTO DENERVATED AREAS OF SKIN
}

\author{
BY \\ G. WEDDELL, L. GUTTMANN AND E. GUTMANN \\ (RECEIVED 18Th November, 1941) \\ Departments of Anatomy, Zoology, and Surgery, University of Oxford \\ Introduction
}

THE existence of extensive overlap between the cutaneous nerve fibres derived from successive nerve roots was first demonstrated by Sherrington (1893) in a study of residual sensibility after section of one or more roots. Other methods, such as that involving local strychninization of the dorsal spinal roots employed by Dusser de Barenne (1910), have been found to give essentially similar results. The method of antidromic stimulation (originally used by Stricker (1876) and Bayliss (1901)) and the study of residual sensibility after the section of dorsal nerve roots were both employed by Foerster (1936) to determine the extent of each human dermatome, and again the correspondence between the results obtained by both methods was close.

The amount of overlap which exists between the peripheral cutaneous nerves of the limbs has been determined by a number of methods, which include anatomical dissection, the successive anæsthetization of nerves supplying contiguous areas of skin followed by sensory tests (Woollard et al., 1940), electrical stimulation methods through the intact skin (Thompson et al., 1934), and finally by the analysis of the sensory loss following peripheral nerve injuries in a large series of patients (Foerster, 1917, 1929). The correspondence between the stimulation and anæsthetization methods is reasonably close. On the other hand, the analysis of sensory loss and sweat secretion (Guttmann, 1940) following nerve injuries shows in many cases very large degrees of overlap which are not demonstrated by the other methods. This finding, taken in conjunction with the clinical observation by Pollock (1920) that the areas of sensory loss following nerve injuries shrink circumferentially before any regeneration can have taken place from the central stump, suggests either that the method of recording residual sensibility is not entirely accurate or that a proportion of overlapping nerve fibres do not transmit impulses giving rise to sensations for some time following a nerve lesion (Pollock, 1919). It is also possible that a new growth of fibres from surrounding normal nerves towards the area of sensory loss takes place (Schuh, 1911, and Weddell and Glees, 1941).

The method of staining nerve fibres throughout large whole preparations of skin by local injection of methylene blue (Weddell, 1941) has been applied 
to experimental animals in an attempt to determine the nature of the shrinkage in the area of sensory loss which follows section of a peripheral nerve before regeneration from the central stump has occurred. Two of us (L. G. and E. G.) have carried out sensory tests (nociceptive) in a large number of rabbits following the division of a variety of peripheral nerves. In general the findings are remarkably constant, and the rabbit as an experimental animal has been found as reliable a subject for observation as man. The data presented here concern the relation of the cutaneous territory of the sural nerve * to those of its adjacent nerves, the tibial and peroneal, and the phenomenon of shrinkage of the area of sensory loss in these areas after resection of either the tibial and peroneal nerves together, or the sural nerve only, before regeneration from the central stump has taken place.

\section{Methods}

Two series of rabbits were employed, in each of which different procedures were adopted.

Series I.-In each of 12 rabbits the following operations were performed. Under intravenous nembutal anæsthesia ( 0.7 c.c. $/$ kilo of a 5 per cent. solution of Abbot's nembutal), and after shaving the skin, the sciatic nerves were exposed with full aseptic precautions in the middle third of each thigh in turn. The sural nerve was dissected away from the tibial and peroneal nerves. On one side the sural nerve was divided, and on the opposite side both the peroneal and tibial nerves were sectioned. Portions of both proximal and distal stumps of each divided nerve were resected to leave as large a gap as possible without damage to the remaining nerve or nerves. Next, the remains of the proximal stumps were turned towards their points of origin, and finally the wounds were closed in two layers with interrupted thread sutures. Very shortly after the operation, and sometimes while the animal was still under light anæsthesia, a sensory test (nociceptive) was carried out. In order to conform as closely as possible to a clinical method commonly used in man, the sensory test consisted of needle pricks with a sharp-pointed, round-bodied, $26 \mathrm{~S}$.W. gauge steel needle, working from the area of sensory loss towards the sensitive skin. The needle point was inserted vertically through the whole thickness of the skin at approximately $1-1 \frac{1}{2}$-second intervals until the first withdrawal reaction occurred; this spot was then marked on the skin with Indian ink. Occasionally the first reaction of the animal to a prick as the sensitive area was approached was a slight increase in the respiratory rate and increased movement of the nares; the next prick (approximately $\frac{1}{2} \mathrm{~mm}$. closer to the sensitive area) was found to cause a definite withdrawal reaction. The first reaction was taken to indicate the boundary of the sensitive area, and this boundary was marked by an ink spot. Immediately following the test, the ink spots which thus outlined the area of sensory loss were tattooed into the skin with an electric tattoo machine. Similar tests were carried out at various intervals following the nerve resection, commencing in many cases on the day following the operation; changes in the area of sensory loss as shown by these tests were also outlined by tattooing ink spots into the skin.

At intervals of 1, 2, 3, 4, and 12 weeks following the first operation, the animals were anæsthetized immediately after a sensory test, and the area of skin supplied by the sural nerve (with a small margin outside) was infiltrated with 0.02 per cent. methylene blue in normal saline solution containing 1 per cent. procaine hydrochloride. This was allowed to stain for three-quarters of an hour, after which the skin and subcutaneous tissues were removed, fixed, and cleared as a total preparation by methods which have been described previously (Weddell, 1941).

\footnotetext{
* Some authors refer to this nerve as the saphenous minor nerve in the rabbit.
} 
After the skin had been removed the animals were killed and the sciatic nerves exposed at the point of operation to see whether any nerve fibres from the proximal stumps had reached the distal stumps of the resected nerves. In doubtful cases portions of intervening tissue were removed and examined microscopically after silver impregnation (see Table 3).

Series II.-In four rabbits, under nembutal anæsthesia, after shaving the skin and with full aseptic precautions, the sciatic nerve was exposed in the middle third of the thigh on the right side only, and the sural nerve separated from the tibial and peroneal nerves as described above; but in these cases none of the nerves was sectioned, although minimal trauma could not be excluded. The wound was closed in two layers as before. Sensory tests (nociceptive) were carried out on the following day, and at intervals of one and two weeks after operation. In these animals sensory tests consisted of a comparison of the withdrawal reactions on the two sides following pin-pricks; in addition, in order to determine whether any motor fibres in the sciatic complex had been damaged, toe-spreading on either side in response to the abrupt lowering of the animal towards the ground was compared. After periods of one week or two weeks following the operation, skin over the outer side of the ankle on both sides was stained with methylene blue and prepared for microscopical examination. The sciatic nerves in their course through the whole of the thigh were also removed with the greatest care, stained in osmic acid, and examined by serial longitudinal sections. The nerves from both sides were treated exactly alike throughout the preparation of the sections. As a further control the sciatic nerve of one rabbit was exposed in the middle third of the thigh on the right side only, but the three separate trunks were not interfered with in any way. The wound was closed in two layers as usual. The sciatic nerves and skin from the ankle regions were likewise removed from this animal for microscopical examination.

\section{Physiological Observations}

After section of the sural nerve only, an area of analgesia, shaped somewhat like a boot, is found to be situated over the lateral aspect of the lower two-thirds

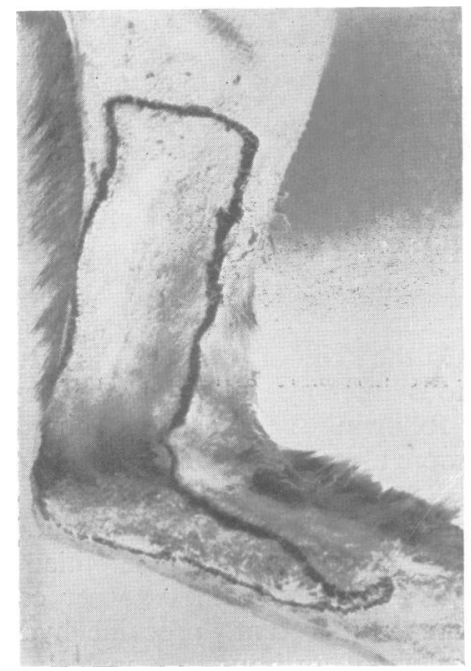

Fig. 1.-The area outlined in Indian in $\mathrm{k}$ is analgetic and was obtained immediately after isolated section of the sural nerve.

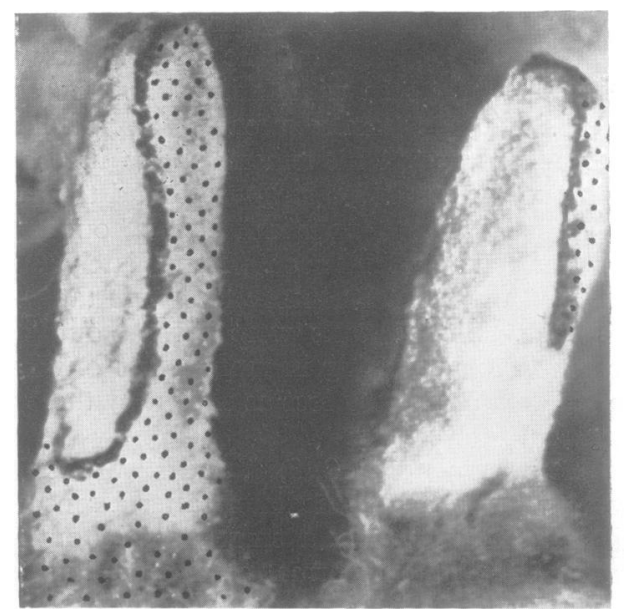

Fig. 2.-The stippled areas are analgetic. The boundaries were obtained immediately after section of the tibial and peroneal nerves on the left side and the sural nerve on the right side. 
of the leg, the lateral part of the ankle, and the proximal part of the lateral margin of the sole and heel (Fig. 1). After section of the tibial and peroneal nerves, the area of remaining sensibility is considerably larger, which indicates the measure of overlap between the sural and its surrounding cutaneous nerves. The overlap is most marked in the sole, where the maximal zone may extend over the greater part of the heel (Fig. 2). The distal extremity of the skin sup plied by the sural nerve on the foot ends in the region of the head of the fifth metatarsal, and here there is considerable overlap (Fig. 3). Since the distal
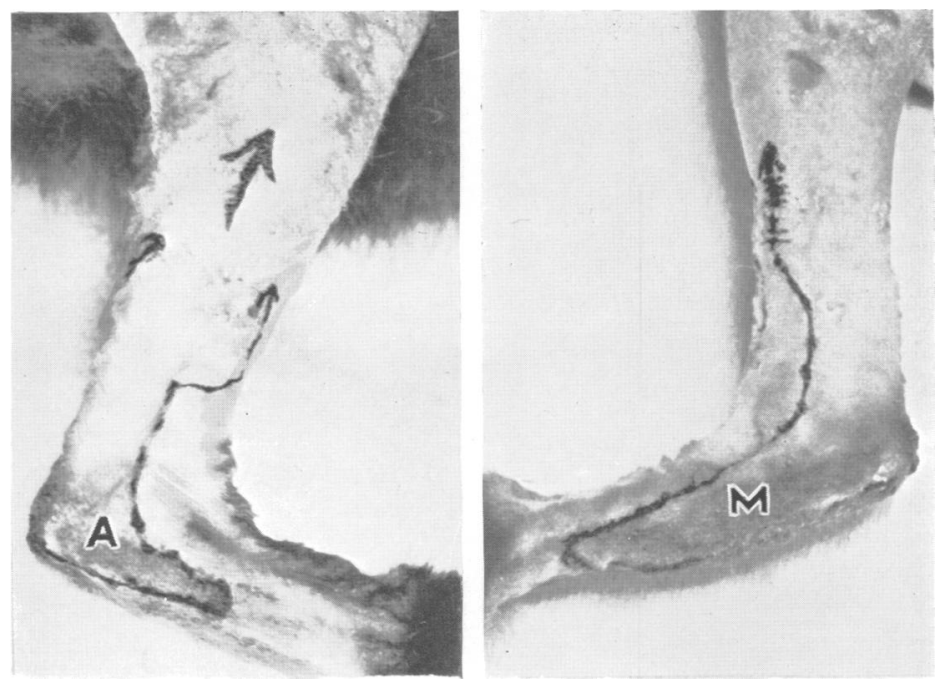

Fig. 3.-The areas outlined were obtained immediately after section of the sural nerve on the left side and the tibial and peroneal nerves on the right side. $\mathrm{A}=$ Centre of autonomous zone; $\mathbf{M}=$ Centre of maximal zone of sural nerve.

extremity of the area of skin supplied by the sural nerve on the foot is surrounded by skin supplied by the peroneal and tibial nerves only, measurements can be taken from the calcaneum to this boundary both when comparing the relative sizes of the autonomous and maximal zones of the sural nerve and when estimating the shrinkage of the area of sensory loss.

Table 1 is a summary of such measurements made on opposite sides of each animal on the day following operation.

Table 1.-Showing Comparison between the Distal Extension of the Maximal and Autonomous Zones of the Sural Nerve

\begin{tabular}{c|c|c}
\hline CASE NO. & AUTONOMOUS ZONE & MAXIMAL ZONE \\
\cline { 2 - 3 } & (millimetres) & (millimetres) \\
9 & 49 & 55 \\
18 & 40 & 54 \\
19 & 43 & 50 \\
22 & 46 & 48 \\
27 & 31 & 44 \\
539 & & 48 \\
\hline
\end{tabular}


A more detailed comparison of the areas of sensory loss on the two sides shows that the overlap from surrounding nerves varies in different regions; in some positions the intermediate zone is large whilst in others it is very much smaller (Fig. 4). The relative size of the intermediate zones remains,

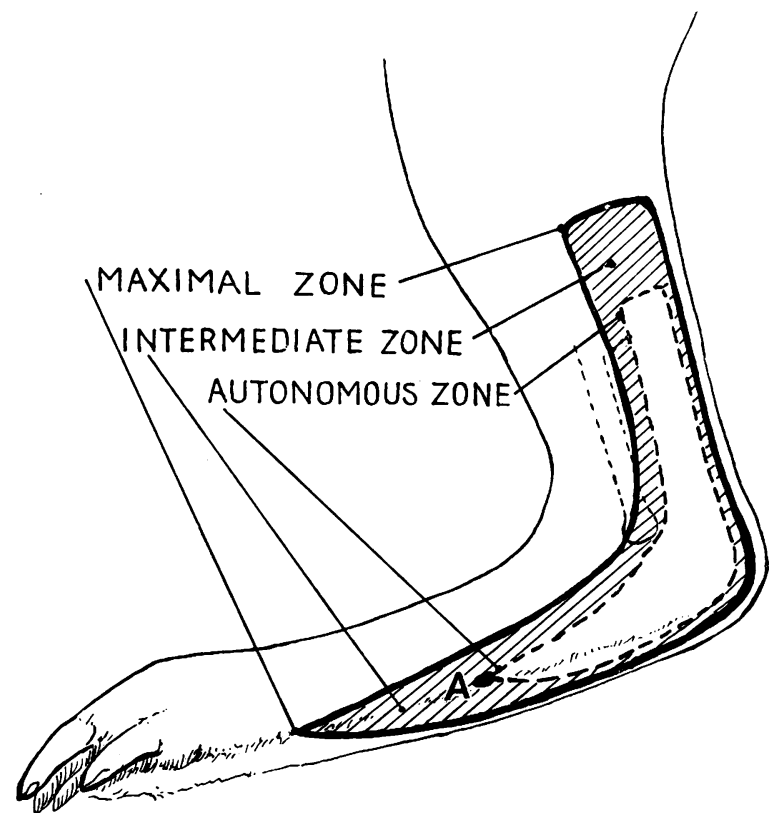

Fig. 4.-Lines representing the outline of both autonomous and maximal zones of the sural nerve have been projected on to a single leg in order to indicate the extent of the intermediate zones. $\quad \mathrm{A}=$ base of 5 th metatarsai.

however, fairly constant from animal to animal. The intermediate zones are always characterized by a diminished number of spots from which nociceptive responses can be aroused; moreover, the responses are diminished in intensity and often delayed as compared with responses from normal areas of skin. In addition to pin-pricks, faradic stimuli were applied through bipolar electrodes in intermediate zones; the threshold was found in all cases to be raised, but more towards the area of sensory loss than close to normal skin (Table 2). Owing to the diminished number of spots from which sensory responses can be aroused, the boundary line between analgetic and normal skin does not appear to be so sharp as compared with areas in which there is a minimum of overlap.

Table 2.--Nociceptive Reactions in the Area supplied by the Sural Nerve after Resection of Tibial and Peroneal Nerves

\begin{tabular}{ll|l|l}
\hline \multicolumn{1}{c|}{ POSITION OF STIMULUS } & FARADIC STIMULUS & \multicolumn{1}{c}{ RESPONSE } \\
\hline Distal extremity of sensitive zone & $\ldots$ & Coil $6 \cdot 5 \mathrm{~cm}$. & $\begin{array}{l}\text { Diminished and sometimes } \\
\text { delayed. } \\
\text { Diminished. } \\
\text { Brisk, maximal. }\end{array}$ \\
$\begin{array}{l}\text { Level of base of fifth metatarsal } \\
\text { Just below external malleolus. }\end{array}$ & $\ldots$ &, $7 \cdot 2-10.3 \mathrm{~cm}$. &, $11 \mathrm{~cm}$.
\end{tabular}


There was never at any time any excessive nociceptive response in rabbits comparable with that which can occasionally be aroused from intermediate zones in man.

Diminution of the Area of Sensory Loss.-Shrinkage of the area of sensory loss was found to take place after isolated section of the sural nerve. The shrinkage is most marked in the neighbourhood of extensive intermediate zones, but is not entirely confined to such areas. The results of sensory tests 1 and 17 days following isolated section of the sural nerve are seen in Fig. 5.

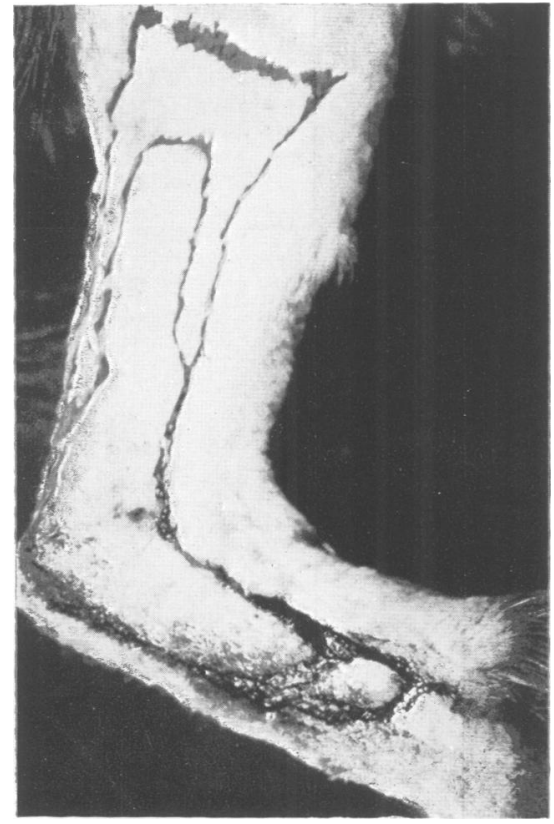

Fig. 5 shows the outline of the areas of sensory loss 1 and 17 days after isolated section of the sural.

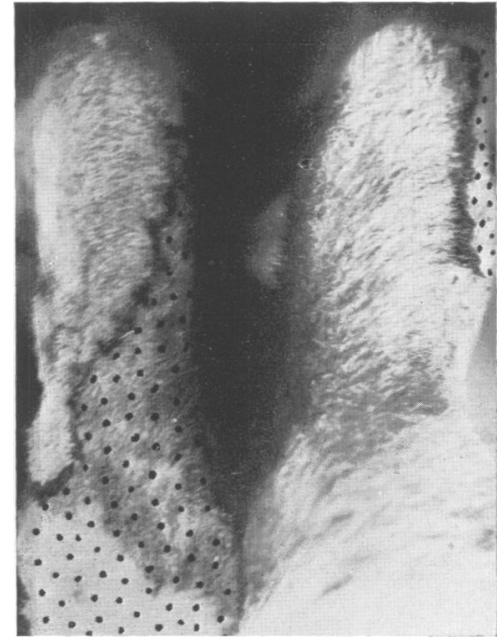

Fig. 6 shows the outline of the areas of sensory loss in the sole $i$ and 17 days after isolated section of the sural nerve. (Compare fig. 2.) The stippled areas are analgetic.

The shrinkage commences in some cases as early as the day following operation (Table 3). It is also clear that following section of the tibial and peroneal nerves there is a steady extension of the maximal zone of the sural nerve; the amount of the extension in one case 17 days after section of the tibial and peroneal nerves is shown in Fig. 6. The character of the response to pin-prick within such zones of shrinkage or extension is in every case similar to that obtained in intermediate zones immediately following operation.

In order to compare the changes in the extent of the autonomous and maximal zones of the sural nerve at various intervals following operation, measurements from the calcaneum to the distal boundary marking the limit of the area of sensory change have been tabulated (Table 3).

In rabbit No. 539 nerve fibres from the proximal stump of the sural nerve had reached the distal stump and regeneration through the distal stump had 
taken place. The rapid shrinkage in the area of sensory loss between the 84th and 91st days presumably coincided with the arrival of fibres from the proximal stump.

Table 3.-Changes in the Extent of the Autonomous and Maximal Zone of the Sural Nerve. Measurements from the Calcaneum to the Distal Boundary of Sensory Change

\begin{tabular}{c|r|r|r|r|r}
\hline & & & \\
& & & \\
NERVE \\
RABBIT \\
NO.
\end{tabular}

In the rabbits in which the sciatic nerve had been exposed on the right side only, and the sural nerve merely separated from the tibial and peroneal nerves, sensory tests (nociceptive) on the day following operation showed that there were a diminished number of spots from which withdrawal reactions could be obtained over the area of sciatic distribution on the operated side as compared with the normal side. In addition the toe-spreading reaction on the operated side was not as wide as that on the unoperated side, suggesting that some motor fibres had also been damaged. One week after operation the changes were far less marked, and at the end of two weeks less marked still, though in all cases some slight difference between the two sides could be detected in favour of the unoperated side. There was great individual variation between the results obtained-in different animals in this series, so that it was not possible to make a statistical analysis. In the one animal in which the sciatic nerve had only been exposed, however, no difference in sensory responses or toe-spreading 
reactions could be detected between the two sides, either on the day following or one and two weeks after operation.

\section{Histological Observations}

Series 1. Maximal Zone of the Sural Nerve.-The pattern of innervation in the area of skin supplied by the nerve was found to bear a general resemblance to that already described in the ear of the rabbit (Weddell, 1941). There is centrally placed a proximo-distally running nerve trunk, from which spring subsidiary nerve trunks, giving rise to bundles which scatter in all directions beneath the skin to enter the cutaneous nerve plexus. As in the case of the

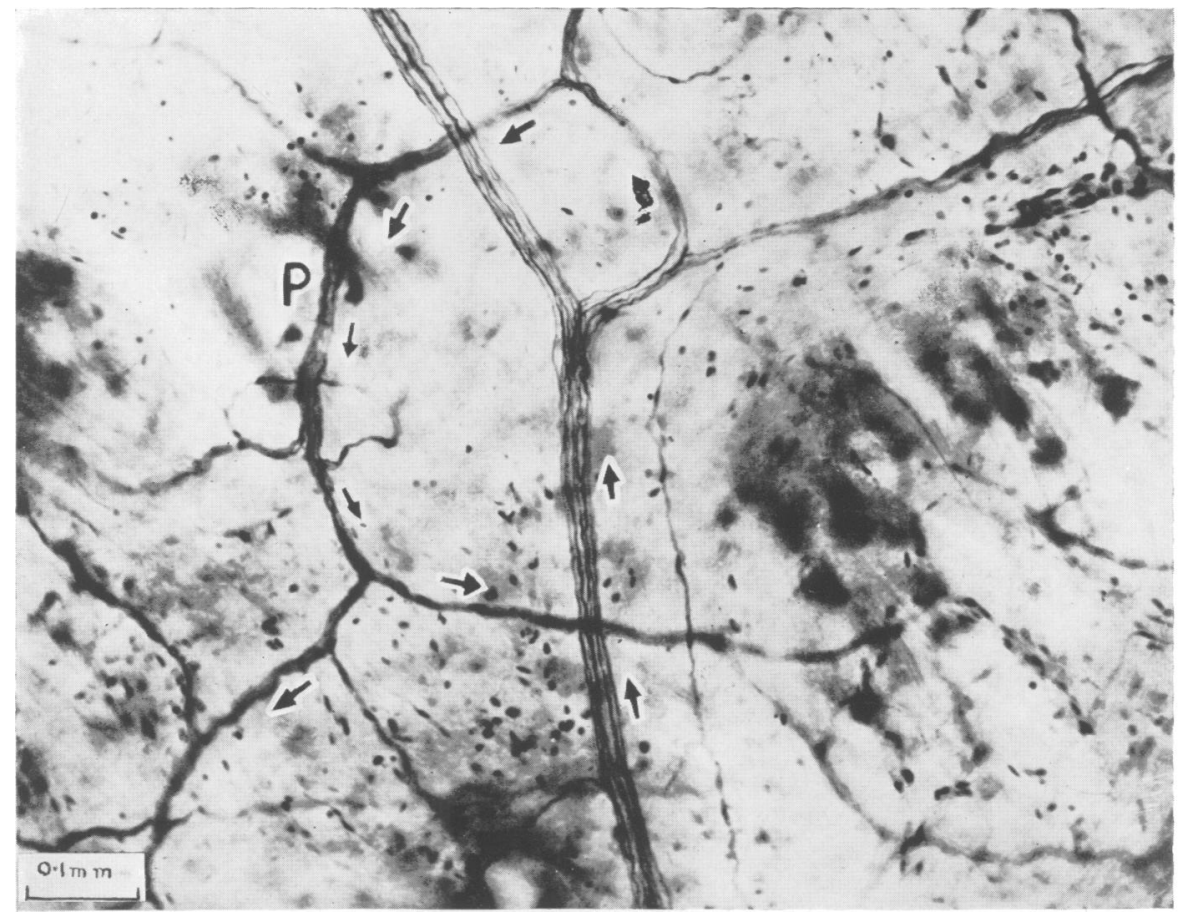

Fig. 7 shows a peripherally running nerve bundle within the maximal zone of the sural nerve which gives rise to fibres entering the cutaneous nerve plexus, the majority of which proceed centrally and superficially towards the skin. The arrows indicate the direction along which the fibres from the nerve trunk are passing. $P=$ cutaneous nerve plexus. The illustration is from a methylene blue stained preparation taken from the skin of the leg of a rabbit.

ear, a large number of nerve fibres pass directly to the periphery of the area of skin which they collectively supply before giving rise to fibres which enter the cutaneous nerve plexus and run centrally and superficially towards the skin. In the illustration (Fig. 7) a peripherally running nerve bundle is shown which gives rise to fibres entering the cutaneous nerve plexus and proceeding centrally and superficially towards the skin.

In skin taken from the centre of the maximal zone supplied by the sural nerve one week after section of the tibial and peroneal nerves, there are sometimes as many as twice the number of nerve fibres undergoing degenerative 
changes as compared with the skin from the same region of an unoperated animal. Apart from this no morphological changes could be detected. In skin removed from similar areas 3, 4, and 12 weeks after section of the tibial and peroneal nerves, no differences could be seen as compared with skin taken from the same region in an unoperated animal. The general neurohistological picture in the skin of the leg is, however, different from that seen in the ear of the rabbit (Fig. 8). In the leg the great majority of hair follicles are supplied by fibres which lose their medullary sheaths during their passage through the cutaneous nerve plexus. At wide intervals of $\frac{1}{2} \mathrm{~cm}$. or more, however, there are large, thick hairs which are supplied by more than one medullated nerve

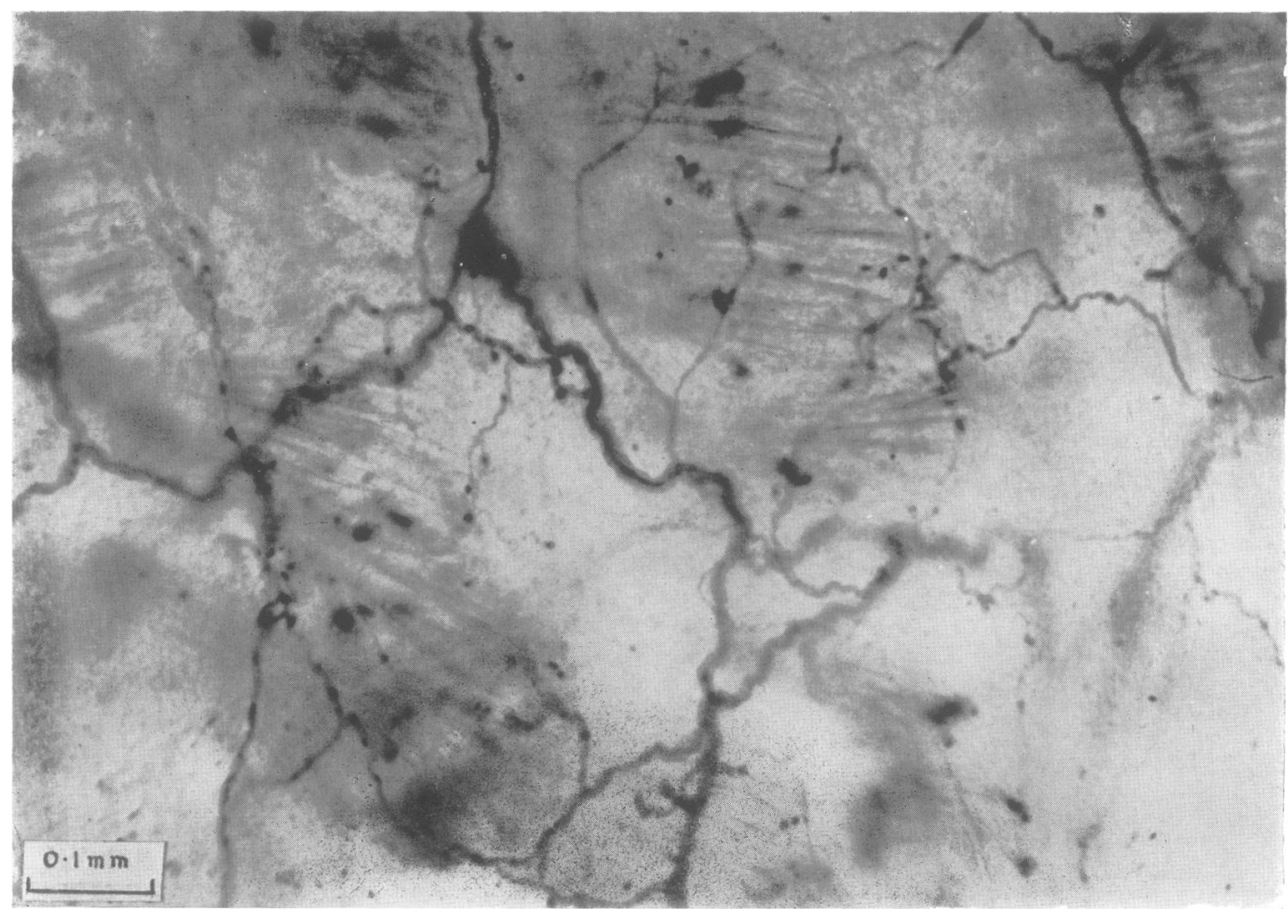

Fig. 8 shows the neurohistology of the centre of the maximal zone of the sural nerve. The staining of the Schwann sheaths and nuclei is clearly seen. Methylene blue stained preparation taken from the skin of the leg of a rabbit.

fibre. The superficial nerve nets which are situated immediately beneath the epidermis, and from which arise fine beaded terminals ending between and beneath the cells of the deepest layers of the epithelium, are similar to those seen in skin from the ear of the rabbit, and a similar interlocking between the nets is apparent. The fact that the majority of fibres of the cutaneous nerve plexus are non-medullated can be readily seen in these preparations because the blue staining of the Schwann sheath and nuclei which invariably occurs after methylene blue staining in this region makes it possible to affirm the absence of a medullary sheath (Figs. 8 and 9). The axis cylinders within the sheaths are often so fine that, owing to the deep staining of the sheath, a proportion 
of them can no longer be seen. Before entering and on leaving the cutaneous nerve plexus, however, these fine nerve fibres are clearly evident, for the Schwann sheaths and nuclei surrounding both the nerve fibres composing the nerve bundles in the subcutaneous tissues, and the fibres emerging from

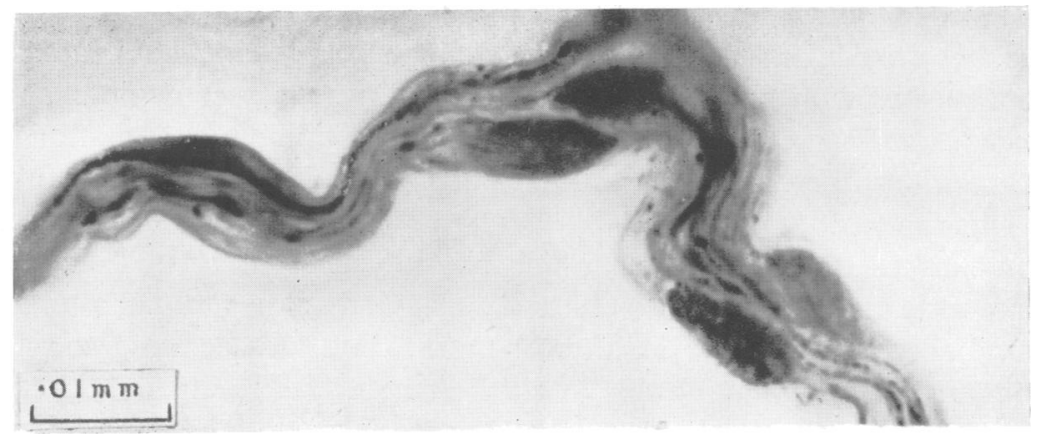

Fig. 9 shows several non-medullated nerve fibres which appear to lie within a single stained sheath; the Schwann nuclei can be clearly seen. The fibres depicted lie in skin from an intermediate zone. Methylene blue stained preparation taken from the skin of the leg of a rabbit.

the plexus and passing towards their terminations, are not stained. This absence of medullation in the great majority of nerve fibres composing the

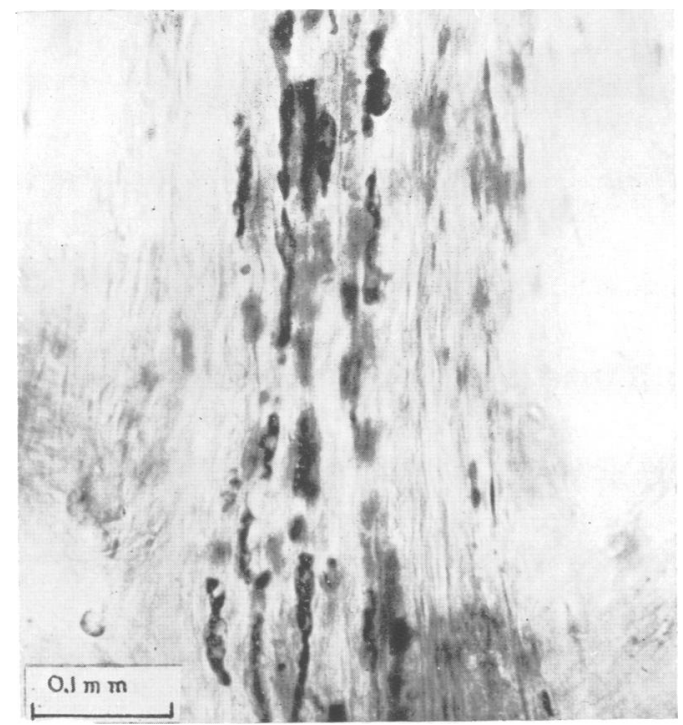

Fig. 10 is from a methylene blue preparation of skin from the leg of a rabbit taken from the centre of the autonomous zone one week after isolated section of the sural nerve. All nerve fibres are seen to be undergoing degenerative changes.

cutaneous nerve plexus has been checked by staining with a modified SpielmeyerHortega method (Weddell and Glees, 1941).

Autonomous Zone of the Sural Nerve.-In skin taken from the centre of the area supplied by the sural nerve one week after its section all the nerve fibres are seen to be undergoing degenerative changes (Fig. 10). In skin taken two 
weeks after section of the nerve, only a few remnants of degenerating nerve fibres are seen. At longer intervals after nerve section no neural remains of any sort are stained by methylene blue.

In a recent study of the early changes in the degeneration of cutaneous nerve fibres (Weddell and Glees, 1941). it has been shown that degenerative changes can be recognized with methylene blue staining as early as 12 hours after nerve section. "Resistant fibres" (Cajal, 1928), however, may show no change for some days after nerve section. For this reason a minimum period of one week was chosen, in order that no "resistant fibres" should be classed as normal in these experiments.

Intermediate Zones.-In skin which physiologically is classed as an intermediate zone and is adjacent to either the maximal or the autonomous zone of the sural nerve taken one week after operation, both degenerating and normal nerve fibres are seen running side by side in nerve bundles situated beneath the skin (Fig. 11). At the level of the cutaneous nerve plexus, however, all

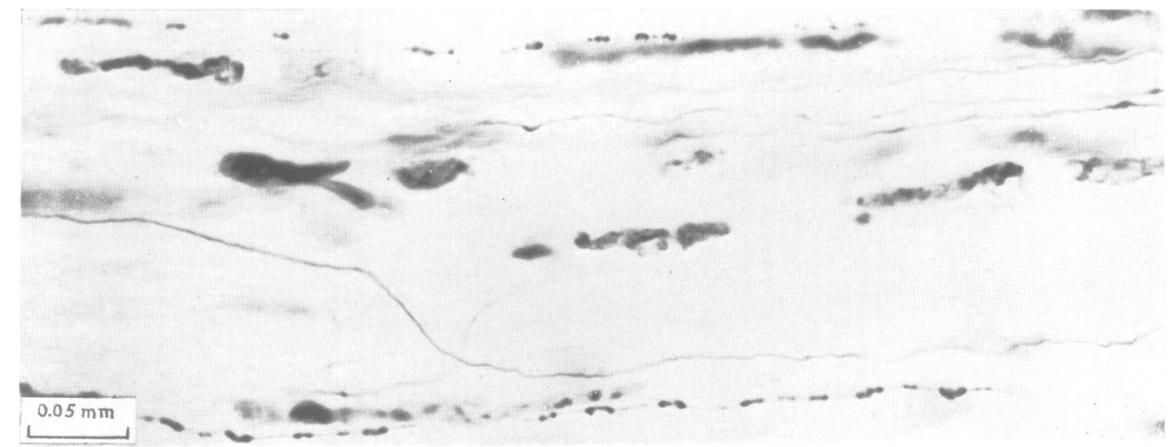

Fig. 11 is from a methylene blue preparation of skin from the leg of a rabbit taken from an intermediate zone one week after isolated section of the sural nerve. Normal and degenerating fibres can be seen lying side by side in a small subcutaneous nerve bundle.

damaged fibres have disappeared, and only those remain which are derived from the intact nerves. The remaining fibres supply hair follicles and give rise to superficially placed nerve nets; the hair follicles, however, are no longer supplied by more than one nerve fibre and the nerve nets are isolated and do not interlock with one another (Fig. 12). The number of nerve fibres in intermediate zones is greatest towards the normal skin and slowly decreases towards the area of sensory loss. The isolated nerve nets invariably extend further towards the denervated areas than the fibres which supply hair follicles. This extension varies in different regions, but reaches a maximum of $2.5 \mathrm{~mm}$ : in the areas of the greatest overlap. In such areas the distance between the nets is about half this distance. It is interesting to note here that stimulation of subepithelial nerve nets by needle-pricks in the ear of the rabbit has been proved to evoke nociceptive responses (Woollard et al., 1940).

Growth of Fibres towards the Area of Sensory Loss.-In skin removed at intervals of 2, 3, 4, and 12 weeks following nerve section, and after shrinkage of the area of sensory loss has taken place, it is found that the neurohistological boundary corresponds extremely closely to the boundary obtained physio- 


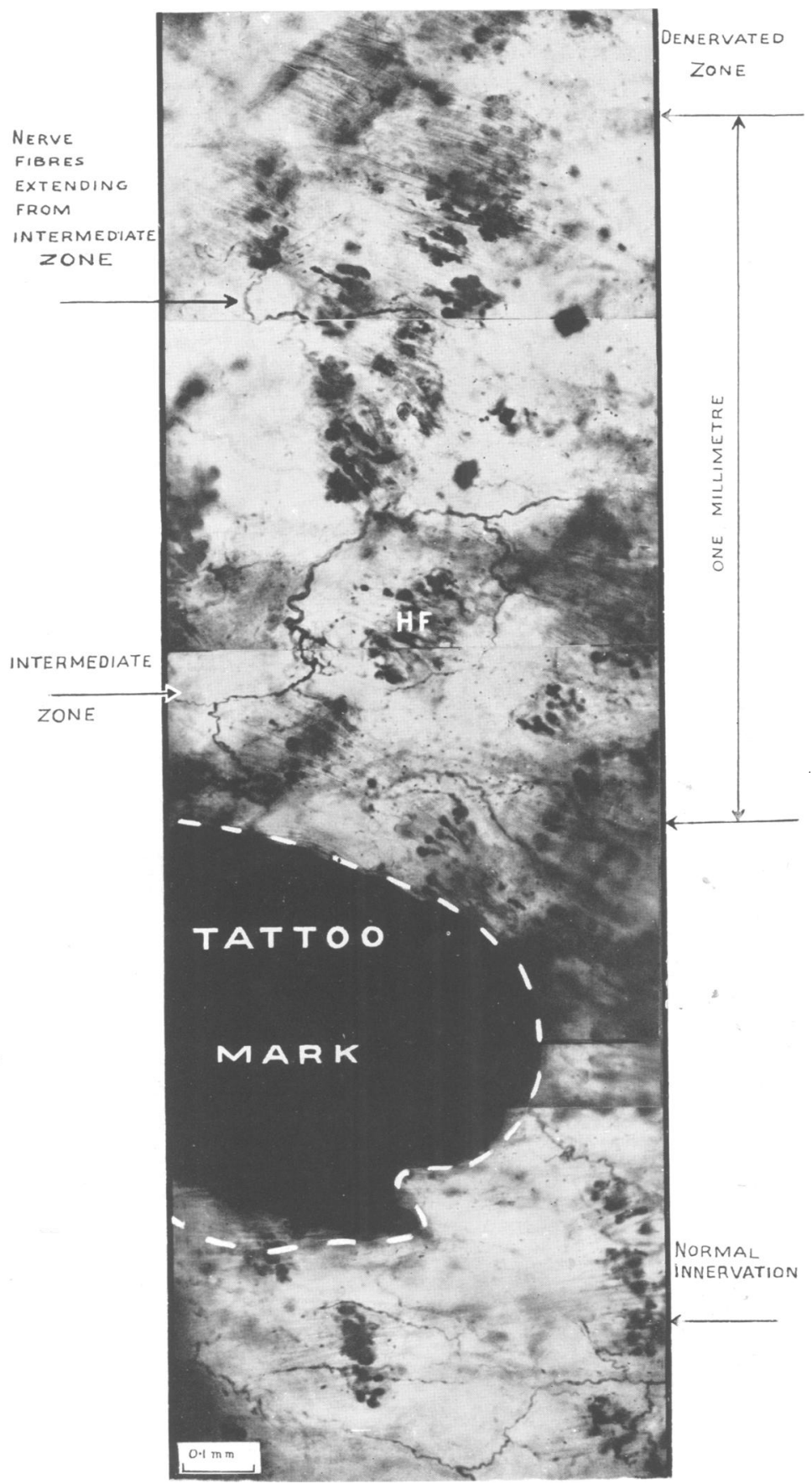

Fig. 12 is from a methylene blue preparation of skin close to the boundary between the maximal zone of the sural nerve and the surrounding denervated skin removed two weeks after section of the tibial and peroneal nerves in a rabbit. It shows the relation between the physiological and neurohistological boundaries. The ink spot represents the physiological boundary, and was tattooed into the skin immediately before staining. Some nerve fibres can be seen extending towards the area of sensory loss. H.F.=Group of hair follicles. 
logically and outlined by the tattoo marks. In the majority of cases, the greatest distance between the boundary marked by the tattoo spots and the boundary of the area shown histologically to contain nerve fibres is $2 \mathrm{~mm}$., whilst the average distance is $1 \mathrm{~mm}$. (Fig. 12). Moreover, the neurohistological boundary corresponds within these limits in each case with the ink dots made just before the removal of the skin; ink dots made at previous tests, but which no longer correspond in position to a sensory boundary, are always found to be surrounded by nerve fibres. The great majority of the nerve fibres between the rows of ink spots demarcating the successive boundaries of the areas of sensory loss are non-myelinated; some supply hair follicles, but most of them

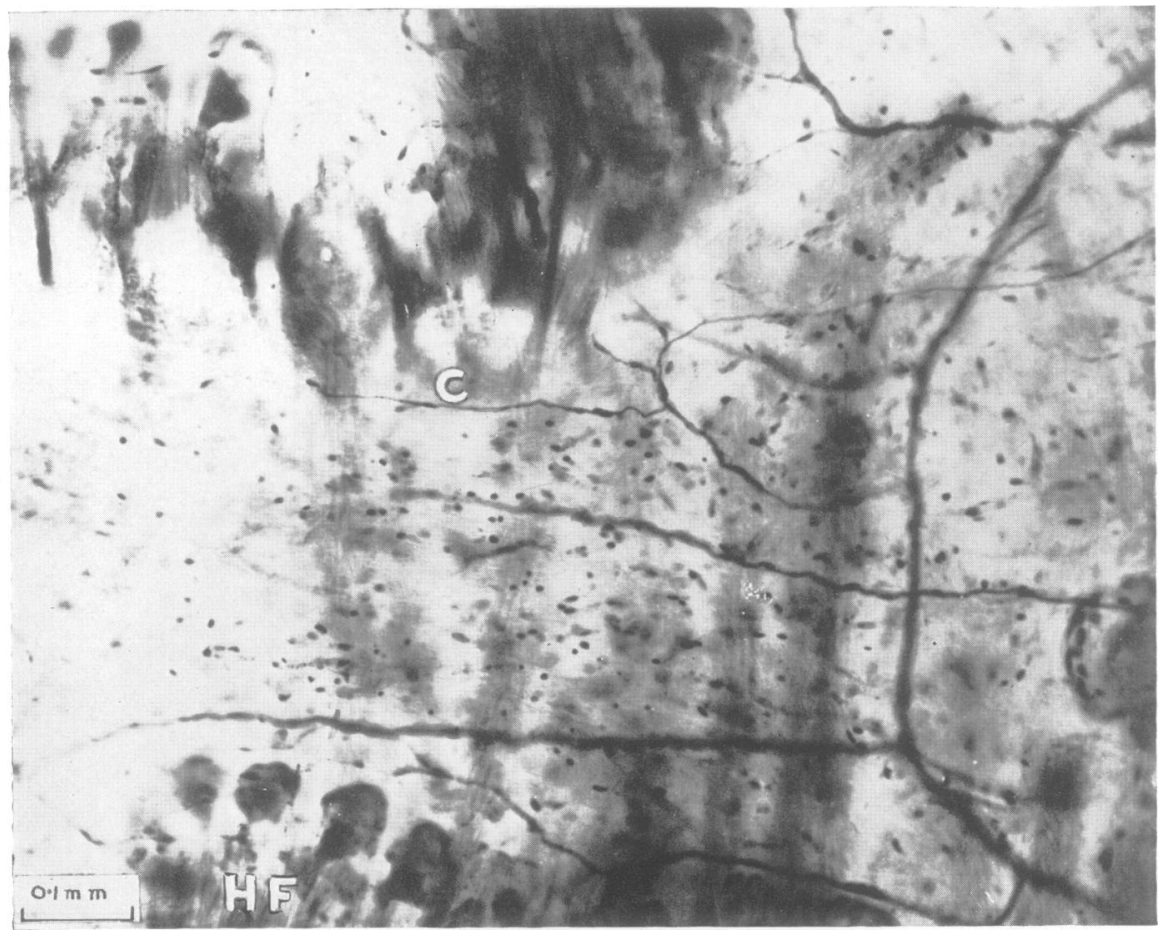

Fig. 13 shows the reorientation of nerve fibres which pass towards the area of sensory loss The fibre marked $\mathrm{C}$, which has a growth cone at its termination, is non-medullated and clearly derived from a fibre which proximally terminates around a hair. The fact that the nerve fibres are advancing from the periphery of an intermediate zone is demonstrated by the solitary nerve fibres which innervate the hairs. H.F.=Group of hair follicles. The illustration is from a methylene blue stained preparation taken from the skin of the leg of a rabbit.

are in the form of fine naked nerve fibres, sometimes beaded and arranged in the form of nets, but more often terminating so finely that they can no longer be traced. These fine nerve fibres are always closer to the area of sensory loss than the fibres supplying the hair follicles. The follicles are not innervated by more than one nerve fibre and there is no clear interlock between the nets which are present.

The advancing nerve fibres become reorientated, so that they appear to be growing towards the area of sensory loss (Fig. 13). Histologically it is very difficult to formulate the characteristic features presented by a growing or 
regenerating nerve fibre. The majority of nerve fibres which have undergone reorientation end in finely beaded or unbeaded terminals, which get progressively finer, until they can no longer be seen. Growth cones are not commonly present at the termination of these fibres. There are, however, a larger number of such cones present in these areas as compared with those which can be found in normal skin. Growth cones are seen at the end of fibres which come from two different sources. Fibres of one type arise from nerve nets or from fibres innervating hair follicles in intermediate zones and pass directly towards the area of sensory loss (Fig. 14). The more rare type of ingrowing fibre which bears a growth cone can be traced for some distance through the cutaneous nerve plexus and subsidiary nerve bundles of the surrounding normal nerves. Such fibres are more deeply situated and occasionally give rise to branches

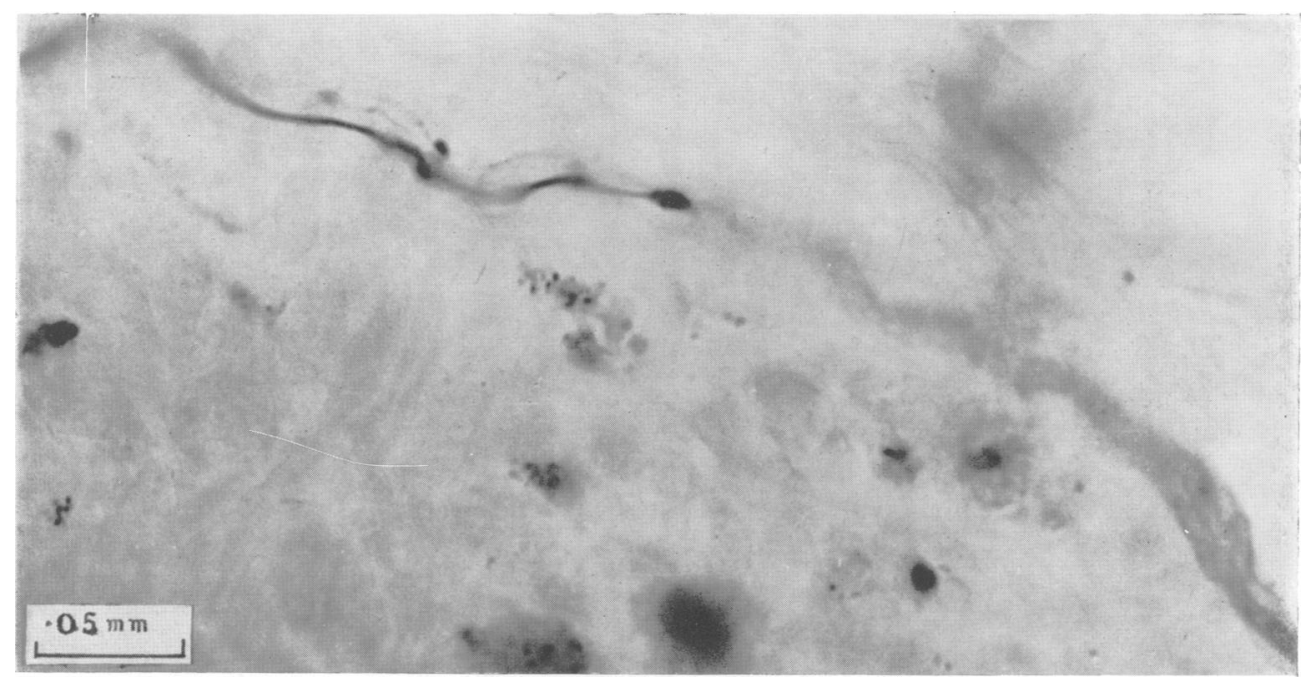

Fig. 14 is taken from skin removed 3 weeks after isolated section of the sural nerve. It shows a growth cone surmounting a nerve fibre advancing towards the area of sensory loss from an intermediate zone. The fibre arises from one which itself gives rise to a subcutaneous nerve net. The outline of the sheath into which the fibre is advancing is clearly seen.

which, however, do not proceed to terminal nets or hairs, but either themselves end in growth cones, or become so fine that they pass beyond the limits of visibility (Fig. 15). Ahead of the growth cones there is always seen a faint blue outline of the sheath into which the cones are advancing. The fibres of the first type are only seen extending from intermediate zones. Fibres of the second type are seen at all points around the periphery of the area of sensory loss. In addition, the fibres of the second type are more abundant in the skin taken from the animal 4 weeks after operation as compared with skin taken at shorter intervals following nerve section. This type of fibre is still more abundant in the skin taken from the animal 12 weeks after nerve section; in this case, however, regeneration from the proximal stump had commenced.

Series II. Exposure of the Sciatic Complex without Nerve Section.-In skin removed from the lateral aspect of the ankle of these rabbits one and two weeks 
after operation, there are about twice the number of degenerating nerve fibres seen on the operated side as compared with the unoperated side. The individual variations are great, but in every case there are more degenerating nerve fibres

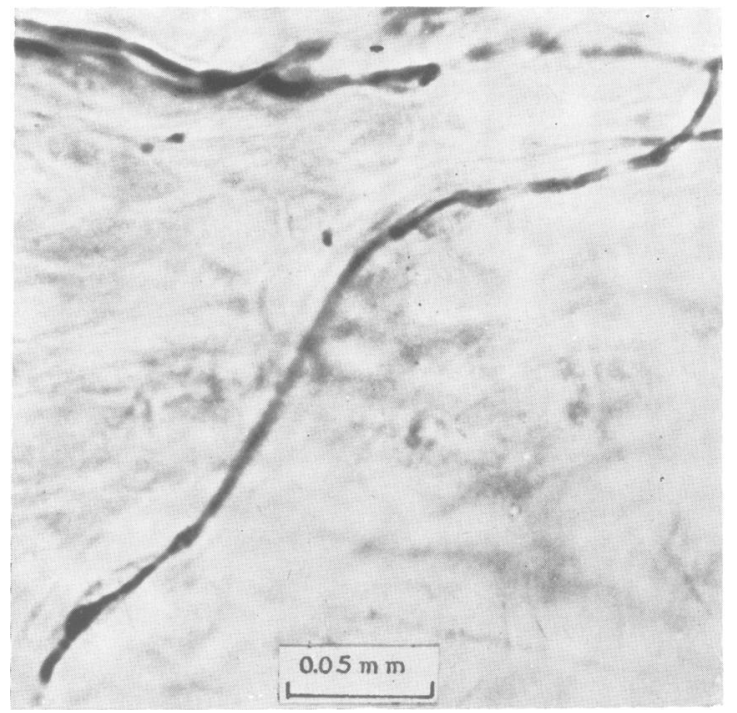

Fig. 15 is from a methylene blue preparation of skin from an area of minimal overlap from the !eg of a rabbit removed 4 weeks after isolated section of the sural nerve. It shows a growth cone surmounting a nerve fibre which can be seen entering an empty sheath. The fibre can be traced into a nerve bundle belonging to the adjacent tibial nerve.

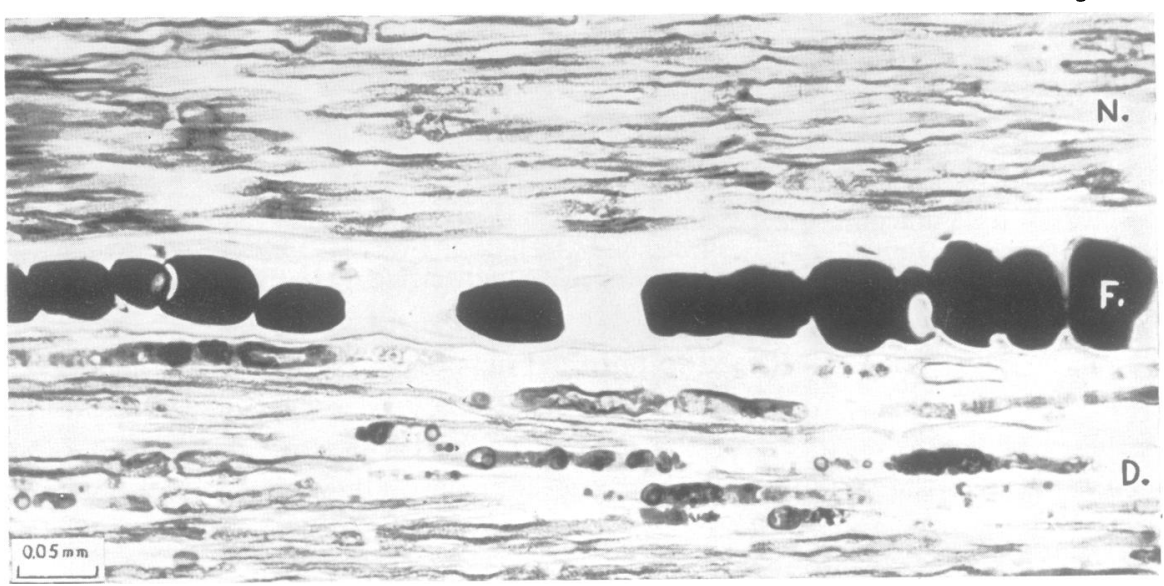

Fig. 16 is from a longitudinal section of the sciatic nerve of a rabbit removed one week after operation. In this case the tibial, peroneal, and sural nerves had merely been dissected free from one another. It shows degenerative changes in one fasciculus only of the sciatic complex. The changes were confined to the nerve below the point of operative interference. Osmic acid stain. $\mathrm{N}=$ Normal fibres; $\mathrm{D}=$ degenerating fibres; $\mathrm{F}=$ fat.

present on the operated side. In the control animal there is no difference between the two sides. In the sciatic nerves there are similarly a larger number of degenerating fibres on the operated side. The degenerating fibres are usually 
confined to bundles on one side of the periphery of the sciatic complex, and occasionally a single fasciculus only is affected; moreover, degeneration is only seen in the sciatic nerves below the level of operative interference (Fig. 16). In the control animal no difference can be detected between the sciatic nerves on either side.

\section{Correlation between the Physiological and Histological Observations}

The physiological observation that no reactions can be aroused by stimulation within the autonomous zone of the cut sural nerve is paralleled by the histological observation that no nerve fibres extend into such an area. It is also clear that no nerve fibres extend beyond the physiological boundary of the maximal zone of the nerve. There is thus no evidence in the rabbit that after nerve section fibres remain in the apparently denervated area of skin which do not give rise to physiological reactions upon stimulation. It is true, however, that no ink dots were found to be placed at the precise distal extremity of the neural boundary, but always just within it, suggesting that stimulation of a single nerve fibre is insufficient to provoke a withdrawal reaction. Intermediate zones from which fewer, diminished, and often delayed reactions could be obtained possessed a diminished number of nerve fibres which no longer gave rise to endings interdigitating with one another, but on the contrary were clearly separated from each other. The histological findings explain the fewer and diminished physiological reactions which can be aroused, but the cause for the delay is not quite so obvious. It is probably explicable on the basis that a " near miss" of the needle point can occur in an intermediate zone owing to the diminished number of endings present. Such a stimulus may give rise to tissue injury, whose products rapidly spread to nerve terminals which will be stimulated.

The correspondence between the boundary obtained physiologically and that seen histologically proves that an actual extension of nerve fibres towards the area of sensory loss takes place in the absence of regeneration from the proximal stump of the divided nerve. It also becomes clear, after reference to Table 3, that the terms "autonomous," "maximal," and "intermediate" zones have a kinetic as opposed to a static anatomical meaning, for directly after nerve section processes commence which alter the extent of these zones.

\section{Discussion}

It is clear from the results recorded above that, after isolated nerve section of the sural nerve, there is a shrinkage in the area of sensory loss due to an ingrowth of nerve fibres from surrounding normal nerves. It is also clear that there is an extension of the maximal zone of the nerve following the section of the tibial and peroneal nerves due to a similar process. This process is a comparatively slow one, as indicated by Table 3 , far slower than would be expected in a process of regeneration from the central stump of the interrupted nerve; moreover, it commences before regeneration could possibly have occurred from above. In any case the possibility of regeneration has been 
excluded in all but one case. ${ }^{*}$ There is evidence that the source of the fibres passing towards the area of sensory loss is twofold. The majority of the fibres which extend from intermediate zones are non-medullated and spring from nerve fibres which either give rise to nerve nets or supply hairs at the periphery of the area of skin innervated. From other than intermediate zones the number of ingrowing fibres is far smaller, and most of those seen advancing towards the area of sensory loss can only be followed proximally into nerve bundles of the surrounding normal nerves. The latter fibres in all probability arise in two different ways. A minor proportion may come from those fibres which in small numbers are certainly found in process of regeneration in normal nerve trunks (a fact to which attention has been drawn in a recent publication-Weddell and Glees, 1941), and in the later stages others are derived from the regeneration of fibres down the surrounding nerve trunks which (it has been demonstrated in the present investigation) are unavoidably damaged during the course of operation unless the most extreme precautions are taken. In other words, the area of sensory loss may be to a varying degree exaggerated as a result of damage to the surrounding nerves.

The orientation of the advancing nerve fibres and their growth along the sheaths of the nerves which have already degenerated suggest either that they are being attracted towards the area of sensory loss by the sheath itself (or by products of degeneration within the sheath), or that nerve fibres always tend to enlarge their terminal ramifications and therefore make use of the unimpeded pathways offered by the empty sheaths. Either of these suggestions is consistent with the fact that the extension of nerve fibres is virtually confined to intermediate zones where overlapping fibres often run in common sheaths (Weddell and Glees, 1941). In this connection it is noteworthy that with rare exceptions there is never any overlap across the midline of the body, a fact well exemplified following resection of the fifth cranial nerve in man. That the attracting or impelling force is a feeble one is shown by the fact that the fibres advance slowly (Table 3); in addition, no extension takes place from a normal fibre unless it is running in a sheath which is common to a degenerating fibre, that is, in an intermediate zone. Tello (1911) favoured the view that the Schwann cells or their products are responsible for attracting new outgrowths of nerve fibres, whilst Boeke (1936) inclined to the view that the outgrowth of nerve fibres is determined by a neurodynamic principle. It is of interest that Speidel (1933, 1935), working on living nerves in the tail of the tadpole, has also observed an extension of nerve fibres into a denervated area by sprouts from nearby normal nerves.

The area of skin supplied by the sural nerve in the rabbit is very small, and, indeed, the process of extension of in-growing fibres from neighbouring zones may lead to the almost complete disappearance of the area of sensory loss, as in Case 22 (Table 3). In addition, after isolated section of the sural nerve in three very young animals (4 weeks old) it was found that shrinkage of the

* It is interesting to compare the increase in the rate of shrinkage in this case when the onset of the regeneration may be presumed to have commenced, i.e. between the 84 th and 91st days. 
area of sensory loss was more rapid than in older animals, and the area of analgesia finally disappeared in each case in the absence of regeneration from the proximal stump.

It is interesting to compare the results obtained in such areas with those which are found in man following peripheral nerve interruptions. The observation by Pollock (1920) that there is a shrinkage in the area of sensory loss before regeneration from the proximal stump of the interrupted nerve takes place, and particularly that the shrinkage involves the sensation of pain, is exactly paralleled by the present observations. In man, pain is subserved by nerve fibres which terminate in non-myelinated nets, and in the rabbit it is just such fibres which extend furthest towards the area of sensory loss. The advance of fibres which terminate around hairs is probably not paralleled in man for the reason that, unlike those in the rabbit, these fibres are myelinated and the continuity of the sheaths is not apparent in such cases. Rivers and Head (1908) and Stopford (1922) have suggested that in many cases following nerve injury pain recovers before touch or other cutaneous sensations in man. This statement, however, does not appear to be confirmed in every case, and it is suggested that a local advance of non-myelinated fibres, i.e. pain fibres, towards the area of sensory loss which may take place from intermediate zones before regeneration from above commences has been mistaken for regeneration. Pain appears to precede other sensations in recovery when in fact regeneration in the usual sense has not yet commenced.

Both Foerster's (1929) and Guttmann's (1940) observations with regard to the very large degrees of overlap which may appear following peripheral nerve injuries may also be explained in part by this local process of extension. It is noteworthy that the overlap of pain and sweating in these cases is always conspicuous, and both these functions are subserved by non-myelinated fibres.

The very early shrinkage in the area of sensory loss (Case 22, Table 3) cannot be studied histologically owing to the possible presence of " resistant fibres." However, the correspondence between the boundary determined physiologically and that seen histologically is so close two weeks or longer after the operation that the early shrinkage must have some peripheral basis. The presence of an increased number of degenerating nerve fibres in trunks supplying skin bordering the area of sensory loss suggests that, in addition to those fibres which show signs of complete interruption, there are others incapable of transmitting impulses, but which will recover without undergoing Wallerian degeneration. The very rapid recovery in the case of the rabbits of Series II supports this hypothesis. Such findings are paralleled in clinical cases by transient blocks with rapid recovery which follow compression lesions such as "Saturday night" paralysis.

Slight degrees of damage to nerves which supply skin surrounding an area of sensory loss may only be apparent in intermediate zones where the number of fibres is already reduced, but nevertheless they may give a false impression of the actual size of the autonomous zone of the divided nerve. Recovery of such damaged fibres may also lead to the erroneous impression that recovery along the course of the interrupted nerve is commencing. Such slight damage to 
nerves which lie in close relationship to an interrupted trunk may be supposed to be common in gun-shot wounds or similar traumatic lesions. It is well known that in such lesions a comparatively trivial superficial wound may be accompanied by more widespread damage in deeply situated tissues. In this connection Foerster (1929) found great differences in the areas of sensory loss between cases in which nerves had been severed cleanly and those which followed gun-shot wounds, the areas in the latter usually being far more extensive than in the former. In fact minimal areas of sensory loss only followed nerve section at aseptic operations; moreover, areas of sensory loss diminished more slowly after gun-shot injuries than after less diffuse lesions. Foerster explained his findings by assuming that the nerve trunks adjacent to the severed nerve were liable to suffer a "concussive" effect, particularly in gun-shot injuries. In view of the observation that the diminution in the area of sensory loss may be due to factors which do not involve regeneration of a divided nerve, it is suggested that in doubtful cases the only certain method of determining whether regeneration is taking place is to block the adjacent nerves with local anæsthetic before carrying out sensory tests.

In view of Foerster's findings, and the fact that in this investigation a small number of degenerating fibres were found in the sciatic nerve following a careful operative procedure which merely involved separating the nerve trunks of the sciatic complex from each other, it is suggested that operative procedures on nerves in man demand the most gentle manipulation.

\section{Summary}

1. After section of the sural nerve in the rabbit the outline of the area of analgesia determined by needle-pricks corresponds to the neurohistological boundary almost exactly. Nerve fibres are never seen within the area of analgesia when sufficient time is allowed for all sectioned fibres to degenerate. This area is the so-called autonomous zone of the sural nerve.

2. After section of the tibial and peroneal nerves the outline of the area of remaining sensibility as determined by needle-pricks corresponds with the neurohistological boundary almost exactly. Nerve fibres are never seen within the area of analgesia surrounding this zone when sufficient time is allowed for all sectioned fibres to degenerate. The area of remaining sensibility is the so-called maximal zone of the sural nerve.

3. The difference in the extent of the autonomous and the maximal zones of the sural nerve is the measure of the overlap between this nerve and its surrounding nerves. These areas are the so-called intermediate zones and are characterized by the fact that fewer, diminished, or delayed responses can be aroused from them by needle-pricks. In these areas there is a diminished number of nerve fibres which give rise to endings which no longer interlock with one another, but, on the contrary, are clearly isolated.

4. Following isolated section of the sural nerve there is a shrinkage of the area of sensory loss which commences as early as the day following operation. 
There is similarly an extension of the territory innervated by the sural nerve following section of the tibial and peroneal nerves.

5. The correspondence between the boundary obtained physiologically and that seen histologically proves that an actual extension of nerve fibres towards the area of sensory loss takes place. This extension is greatest from the periphery of intermediate zones, and here the advancing fibres are nonmedullated. These spring from nerve fibres which give rise to nerve nets or which supply hair follicles at the periphery of the area of innervated skin. Elsewhere the number of ingrowing fibres is smaller and the majority can only be followed proximally into nerve bundles of the surrounding normal nerves; some of these fibres are probably derived from the regeneration of fibres down the surrounding nerve trunks (which it has been demonstrated in the present investigation are invariably damaged in the course of operation, even when most careful precautions are taken).

6. The terms " autonomous," " maximal," and " intermediate " zones have a relative as opposed to a static anatomical significance, for directly after nerve section processes commence which alter the extent of these zones.

7. The physiological and histological findings have been discussed in the light of clinical findings.

The expenses of this investigation were defrayed by a grant from the Medical Research Council (G.W.) L. G. wishes gratefully to acknowledge grants by the Nuffield Committee for the Advancement of Medicine and by Balliol College, Oxford.

\section{REFERENCES}

Bayliss, W. M. (1901). J. Physiol., 26, 173.

Cajal, S. R. (1928). Degeneration and Regeneration of the Nervous System. Oxford University Press.

Dusser de Barenne, J. G. (1910). Folia Neuro-Biol., Leipzig, 5, 342.

Boeke, J. (1936). Handb. d. Neurol., by Bumke and Foerster, 1936, Vol. 1, 1053. J. Springer, Berlin.

Foerster, O. (1917). Verhandl. Ges. Dtsch. Nervenaerzte, 1917, 80.

- (1929). Handb. Neurol., by Bumke and Foerster, Errgänzungsband 2, 1025. J. Springer, Berlin. (1936). Ibid., 2nd ed.

Guttmann, L. (1940). J. Neurol. Psychiat., 3, 197.

Pollock, L. J. (1919). Arch. Neurol. Psychiat., 2, 667. (1920). J. comp. Neurol., 32, 357.

Rivers, W. H. R., and Head, H. (1908). Brain, 31, 323.

Sherrington, C. S. (1893). Philos. Trans., 184 B, 641.

Speidel, C. C. (1933). Amer. J. Anat., 52, 1.

(1935). J. comp. Neurol., 61, 1.

Stopford, J. S. B. (1922). Brain, 45, 385.

Stricker, S. (1876). S.B. Akad. Wiss. Wien (Math. Nat. Kl., Abt. 3).

Schuh (quoted by Oppenheim (1911). Textbook on Nervous Diseases, Chicago, 1, 408.

Tello, F. (1911). Trab. Lab. Invest. biol. Univ., Madrit, 9, 123.

Thompson, I. M., Inman, V. T., and Brownfield, B. (1934). Univ. Calif. Publ. Anat., 1, 7.

Weddell, G. (1941). J. Anat., London, 75, 346.'

Weddell, G., and Glees, P. (1941). J. Anat., London, 76, 65.

Woollard, H. H., Weddell, G., and Harpman, J. A. (1940). J. Anat., London, 74, 413. 